\title{
On Weil restriction of reductive groups and a theorem of Prasad
}

\author{
Journal Article
}

Author(s):

Pink, Richard

Publication date:

2004-11

Permanent link:

https://doi.org/10.3929/ethz-b-000160278

Rights / license:

In Copyright - Non-Commercial Use Permitted

Originally published in:

Mathematische Zeitschrift 248(3), https://doi.org/10.1007/s002090100339 


\title{
On Weil restriction of reductive groups and a theorem of Prasad
}

\author{
Richard Pink \\ Departement Mathematik, ETH Zentrum, CH-8092 Zürich, Switzerland \\ (e-mail: pink@math.ethz.ch)
}

Received: 19 October 2000; in final form: 3 January 2001 /

Published online: 31 August 2004 - (C) Springer-Verlag 2004

\begin{abstract}
Let $G$ be a connected simple semisimple algebraic group over a local field $F$ of arbitrary characteristic. In a previous article by the author the Zariski dense compact subgroups of $G(F)$ were classified. In the present paper this information is used to give another proof of a theorem of Prasad [8] (also proved by Margulis [3]) which asserts that, if $G$ is isotropic, every non-discrete closed subgroup of finite covolume contains the image of $\tilde{G}(F)$, where $\tilde{G}$ denotes the universal covering of $G$. This result played a central role in Prasad's proof of strong approximation. The present proof relies on some basic properties of Weil restrictions over possibly inseparable field extensions, which are also proved here.
\end{abstract}

Mathematics Subject Classification (2000): 20G25, 14L15

\section{Weil restriction of linear algebraic groups}

Let $F$ be a field and $F^{\prime}$ a subfield such that $\left[F / F^{\prime}\right]<\infty$. In this section we discuss some properties of the Weil restriction $\mathcal{R}_{F / F^{\prime}} G$ where $G$ is a linear algebraic group over $F$. We are interested particularly in the case that $F / F^{\prime}$ is inseparable, where the Weil restriction involves some infinitesimal aspects. Thus the natural setting is that of group schemes. We assume that $G$ is a connected affine group scheme of finite type that is smooth over $F$. The smoothness condition is equivalent to saying that $G$ is reduced and "defined over $F$ " in the terminology of [11] Ch.11.

Throughout, we will speak of a scheme over a ring $R$ when we really mean a scheme over Spec $R$. Similarly, for any ring homomorphism $R^{\prime} \rightarrow R$ and any scheme $X^{\prime}$ over $R^{\prime}$ we will abbreviate $X^{\prime} \times{ }_{R^{\prime}} R:=X^{\prime} \times{ }_{\operatorname{Spec}} R^{\prime} \operatorname{Spec} R$. The basic facts on Weil restrictions that we need are summarized in [4] Appendix 2-3. Throughout the following we abbreviate

$$
G^{\prime}:=\mathcal{R}_{F / F^{\prime}} G
$$


By [4] A.3.2, A.3.7 this is a connected smooth affine group scheme over $F^{\prime}$. The universal property of the Weil restriction identifies $G^{\prime}\left(F^{\prime}\right)$ with $G(F)$.

Next, we fix an algebraic closure $E^{\prime}$ of $F^{\prime}$ and abbreviate $E:=F \otimes_{F^{\prime}} E^{\prime}$. With $\Sigma:=\operatorname{Hom}_{F^{\prime}}\left(F, E^{\prime}\right)$ there is then a unique decomposition $E=\bigoplus_{\sigma \in \Sigma} E_{\sigma}$, where each $E_{\sigma}$ is a local ring with residue field $E^{\prime}$ and the composite map $F \rightarrow E_{\sigma} \rightarrow E^{\prime}$ is equal to $\sigma$. The Weil restriction from any finite dimensional commutative $E^{\prime}$ algebra down to $E^{\prime}$ is defined, and by [4] A.2.7-8 we have natural isomorphisms

$$
\begin{aligned}
G^{\prime} \times F_{F^{\prime}} E^{\prime} & \cong \mathcal{R}_{E / E^{\prime}}\left(G \times_{F} E\right) \\
& =\mathcal{R}_{E / E^{\prime}}\left(\bigsqcup_{\sigma \in \Sigma} G \times_{F} E_{\sigma}\right) \\
& \cong \prod_{\sigma \in \Sigma} G_{\sigma}
\end{aligned}
$$

with

$$
G_{\sigma}:=\mathcal{R}_{E_{\sigma} / E^{\prime}}\left(G \times{ }_{F} E_{\sigma}\right) .
$$

These isomorphisms are functorial in $G$ and equivariant under $\operatorname{Aut}\left(E^{\prime} / F^{\prime}\right)$, which acts on the right hand side by permuting the factors according to its action on $\Sigma$. Next, for every $\sigma \in \Sigma$ we fix a filtration of $E_{\sigma}$ by ideals

$$
E_{\sigma} \supsetneqq I_{\sigma, 1} \supsetneqq \cdots \supsetneqq I_{\sigma, q-1} \supsetneqq I_{\sigma, q}=0
$$

with subquotients of length 1 . Here $q$ is the degree of the inseparable part of $F / F^{\prime}$. We also choose a basis of every successive subquotient. For every $1 \leq i \leq q$ there is a natural homomorphism

$$
G_{\sigma}=\mathcal{R}_{E_{\sigma} / E^{\prime}}\left(G \times{ }_{F} E_{\sigma}\right) \longrightarrow \mathcal{R}_{\left(E_{\sigma} / I_{\sigma, i}\right) / E^{\prime}}\left(G \times{ }_{F}\left(E_{\sigma} / I_{\sigma, i}\right)\right) .
$$

Let $G_{\sigma, i}$ denote its kernel. By [4] A.3.5 we find that each $G_{\sigma, i}$ is smooth over $F^{\prime}$ and there are canonical isomorphisms

$$
G_{\sigma} / G_{\sigma, 1} \cong G \times_{F, \sigma} E^{\prime}
$$

and

$$
G_{\sigma, i} / G_{\sigma, i+1} \cong \operatorname{Lie} G \otimes_{F, \sigma} \mathbb{G}_{a, E^{\prime}}
$$

for all $1 \leq i \leq q-1$, where $\mathbb{G}_{a}$ denotes the additive group of dimension 1. Moreover, this description is functorial in $G$. Namely, let $H$ be another smooth group scheme over $F$ and define $H^{\prime}:=\mathcal{R}_{F / F^{\prime}} H, H_{\sigma}$ and $H_{\sigma, i}$ in the obvious way. Then any homomorphism $\varphi: H \rightarrow G$ induces homomorphisms $\mathcal{R}_{F / F^{\prime}} \varphi: H^{\prime} \rightarrow G^{\prime}$, $H_{\sigma} \rightarrow G_{\sigma}$ and $H_{\sigma, i} \rightarrow G_{\sigma, i}$ and the resulting homomorphisms on subquotients are just

$$
\varphi \times \mathrm{id}: H \times_{F, \sigma} E^{\prime} \longrightarrow G \times_{F, \sigma} E^{\prime}
$$

and

$$
d \varphi \otimes \text { id : Lie } H \otimes_{F, \sigma} \mathbb{G}_{a, E^{\prime}} \longrightarrow \text { Lie } G \otimes_{F, \sigma} \mathbb{G}_{a, E^{\prime}}
$$


Recall that an isogeny of algebraic groups is a surjective homomorphism with finite kernel. An isogeny $\varphi$ is separable if and only if its derivative $d \varphi$ is an isomorphism.

Proposition 1.6. Let $\varphi: H \rightarrow G$ be a homomorphism of connected smooth linear algebraic groups over $F$.

(a) If $F / F^{\prime}$ is separable, then $\mathcal{R}_{F / F^{\prime}} \varphi: H^{\prime} \rightarrow G^{\prime}$ is an isogeny if and only if $\varphi$ is an isogeny.

(b) If $F / F^{\prime}$ is inseparable, then $\mathcal{R}_{F / F^{\prime}} \varphi: H^{\prime} \rightarrow G^{\prime}$ is an isogeny if and only if $\varphi$ is a separable isogeny.

Proof. In the separable case we have $E^{\prime} \stackrel{\sim}{\longrightarrow} E_{\sigma}$, and assertion (a) follows directly from the decomposition 1.1 and the functoriality 1.4 . So assume that $F / F^{\prime}$ is inseparable, i.e., that $q>1$. First note that $\operatorname{dim} H^{\prime}=\left[F / F^{\prime}\right] \cdot \operatorname{dim} H$ and $\operatorname{dim} G^{\prime}=$ $\left[F / F^{\prime}\right] \cdot \operatorname{dim} G$, by the successive extension above or by [4] A.3.3. Thus if either $\varphi$ or $\mathcal{R}_{F / F^{\prime}} \varphi$ is an isogeny, we must have $\operatorname{dim} H=\operatorname{dim} G$.

If $\mathcal{R}_{F / F^{\prime}} \varphi$ is an isogeny, its kernel is finite; hence so is the kernel of its restriction $H_{\sigma, q-1} \rightarrow G_{\sigma, q-1}$. By 1.5 this means that $d \varphi$ is injective. For dimension reasons it follows that $d \varphi$ is an isomorphism; hence $\varphi$ is a separable isogeny, as desired.

Conversely, suppose that $\varphi$ is a separable isogeny. Then all the homomorphisms on subquotients 1.4 and 1.5 induced by $\mathcal{R}_{F / F^{\prime}} \varphi$ are surjective. Using the snake lemma inductively one deduces that $\mathcal{R}_{F / F^{\prime}} \varphi$ itself is surjective. For dimension reasons it is therefore an isogeny, as desired.

Theorem 1.7. If $G$ is reductive and $F^{\prime}$ infinite, then $G^{\prime}\left(F^{\prime}\right)$ is Zariski dense in $G^{\prime}$.

Proof. If $F / F^{\prime}$ is separable, the isomorphism 1.1 shows that $G^{\prime}$ is reductive. In that case the assertion is well-known: see [11] Cor.13.3.12 (i).

We will adapt the argument to the general case.

Assume first that $G=T$ is a torus. Choose a finite separable extension $F_{1} / F$ which splits $T$, and fix an isomorphism $\mathbb{G}_{m, F_{1}}^{n} \stackrel{\sim}{\longrightarrow} T \times{ }_{F} F_{1}$, where $\mathbb{G}_{m}$ denotes the multiplicative group of dimension 1. Combining this with the norm map yields a surjective homomorphism

$$
\mathcal{R}_{F_{1} / F} \mathbb{G}_{m, F_{1}}^{n} \longrightarrow \mathcal{R}_{F_{1} / F}\left(T \times_{F} F_{1}\right) \stackrel{\mathrm{Nm}}{\longrightarrow} T .
$$

Since $F_{1} / F$ is separable, this morphism is smooth. By [4] A.2.4, A.2.12 it induces a smooth homomorphism

$$
\mathcal{R}_{F_{1} / F^{\prime}} \mathbb{G}_{m, F_{1}}^{n} \cong \mathcal{R}_{F / F^{\prime}} \mathcal{R}_{F_{1} / F} \mathbb{G}_{m, F_{1}}^{n} \longrightarrow \mathcal{R}_{F / F^{\prime}} T
$$

In particular, this morphism is dominant. On the other hand we have an open embed$\operatorname{ding} \mathbb{G}_{m, F_{1}}^{n} \hookrightarrow \mathbb{A}_{F_{1}}^{n}$ and hence, by [4] A.2.11, an open embedding $\mathcal{R}_{F_{1} / F^{\prime}} \mathbb{G}_{m, F_{1}}^{n} \hookrightarrow$ $\mathcal{R}_{F_{1} / F^{\prime}} \mathbb{A}_{F_{1}}^{n}$. It is trivial to show that $\mathcal{R}_{F_{1} / F^{\prime}} \mathbb{A}_{F_{1}}^{n} \cong \mathbb{A}_{F^{\prime}}^{n d}$, where $d=\left[F_{1} / F^{\prime}\right]$. It follows that the $F^{\prime}$-rational points in $\mathcal{R}_{F_{1} / F^{\prime}} \mathbb{G}_{m, F_{1}}^{n}$ are Zariski dense, and so their images form a Zariski dense set of $F^{\prime}$-rational points in $\mathcal{R}_{F / F^{\prime}} T$, proving the theorem in this case.

If $G$ is arbitrary let $T$ be a maximal torus of $G$. As $\mathcal{R}_{F / F^{\prime}} T$ is commutative, it possesses a unique maximal torus $T^{\prime}$, which is smooth over $F^{\prime}$ by [11] Thm.13.3.6. 
Lemma 1.8. $\mathcal{R}_{F / F^{\prime}} T$ is the centralizer of $T^{\prime}$ in $G^{\prime}$.

Proof. If $F / F^{\prime}$ is separable, this follows from the fact that $\mathcal{R}_{F / F^{\prime}} T$ is a maximal torus of $G^{\prime}$. So assume that $F / F^{\prime}$ is inseparable of characteristic $p$. Since $\left(\mathcal{R}_{F / F^{\prime}} T\right) / T^{\prime}$ is unipotent, we have $T^{\prime}=\left(\mathcal{R}_{F / F^{\prime}} T\right)^{p^{n}}$ for suitable $n \gg 0$. As $T^{\prime}$ is smooth and the rational points of $\mathcal{R}_{F / F^{\prime}} T$ are Zariski dense, the centralizer of $T^{\prime}$ is equal to the centralizer of $\left(\mathcal{R}_{F / F^{\prime}} T\right)\left(F^{\prime}\right)^{p^{n}}$. Note that the universal property of the Weil restriction identifies $\left(\mathcal{R}_{F / F^{\prime}} T\right)\left(F^{\prime}\right)$ with $T(F)$.

Consider a scheme $S^{\prime}$ over $F^{\prime}$ and an $S^{\prime}$-valued point $\varphi^{\prime}: S^{\prime} \rightarrow G^{\prime}$. Via the universal property of the Weil restriction $\varphi^{\prime}$ corresponds to an $S^{\prime} \times{ }_{F^{\prime}} F$-valued point $\varphi: S^{\prime} \times{ }_{F^{\prime}} F \rightarrow G$. We have seen that $\varphi^{\prime}$ factors through the centralizer of $T^{\prime}$ if and only if it commutes with $\left(\mathcal{R}_{F / F^{\prime}} T\right)\left(F^{\prime}\right)^{p^{n}}$. This is equivalent to saying that $\varphi$ commutes with $T(F)^{p^{n}}$. As $T$ is a torus and $F$ infinite, the subgroup $T(F)^{p^{n}}$ is Zariski dense in $T$. The condition therefore amounts to saying that $\varphi$ factors through the centralizer of $T$. But this centralizer is equal to $T$. Therefore, translated back to $G^{\prime}$, the condition says that $\varphi^{\prime}$ factors through $\mathcal{R}_{F / F^{\prime}} T$. This proves the lemma.

By Lemma 1.8 the subgroup $\mathcal{R}_{F / F^{\prime}} T$ is the centralizer of a maximal torus of $G^{\prime}$, i.e., it is a Cartan subgroup of $G^{\prime}$. Thus [11] Cor.13.3.12 implies that $G^{\prime}\left(F^{\prime}\right)$ is Zariski dense in $G^{\prime}$, proving Theorem 1.7.

Remark 1.9. If $F^{\prime}$ is a non-discrete complete normed field, Theorem 1.7 is true for arbitrary connected smooth algebraic groups $G$. This is an easy consequence of the implicit function theorem.

Next we turn to simple groups. To fix ideas, a smooth linear algebraic group over a field will be called simple if it is non-trivial and possesses no non-trivial proper connected smooth normal algebraic subgroup. It is called absolutely simple if it remains simple over the algebraic closure of the base field.

If $G$ is simply connected semisimple and simple over $F$, it is isomorphic to $\mathcal{R}_{F_{1} / F} G_{1}$ for an absolutely simple simply connected semisimple group $G_{1}$ over some finite separable extension $F_{1} / F$ (cf. [11] Ex.16.2.9). From [4] A.2.4 we then deduce that $G^{\prime} \cong \mathcal{R}_{F_{1} / F^{\prime}} G_{1}$. In this way questions about $G^{\prime}$ can be reduced to the case that $G$ is absolutely simple.

Theorem 1.10. Assume that $G$ is simply connected semisimple and simple over $F$. Then $G^{\prime}$ is simple over $F^{\prime}$.

Proof. By the above remarks we may assume that $G$ is absolutely simple. Consider a non-trivial connected smooth normal algebraic subgroup $H^{\prime} \subset G^{\prime}$. Let

$$
\bar{H}^{\prime} \subset \prod_{\sigma \in \Sigma} G \times_{F, \sigma} E^{\prime}
$$

denote the image of $H^{\prime} \times{ }_{F^{\prime}} E^{\prime}$ under the composite of the natural maps

$$
G^{\prime} \times_{F^{\prime}} E^{\prime} \stackrel{1.1}{\cong} \prod_{\sigma \in \Sigma} G_{\sigma} \rightarrow \prod_{\sigma \in \Sigma} G_{\sigma} / G_{\sigma, 1} \stackrel{1.2}{\cong} \prod_{\sigma \in \Sigma} G \times_{F, \sigma} E^{\prime}
$$


Since $H^{\prime}$ is non-trivial and "defined over $F^{\prime \prime}$ ", by [11] Cor.12.4.3 we have $\bar{H}^{\prime} \neq 1$. Since $H^{\prime} \subset G^{\prime}$ is a connected normal subgroup, so is $\bar{H}^{\prime}$ in 1.11 . It is therefore equal to the product of some of the factors on the right hand side. As $\bar{H}^{\prime}$ is non-trivial, it contains at least one of these factors. But by construction it is also invariant under $\operatorname{Aut}\left(E^{\prime} / F^{\prime}\right)$, which permutes the factors transitively. We deduce that the inclusion 1.11 is in fact an equality. Now the following lemma implies that $H^{\prime} \times{ }_{F^{\prime}} E^{\prime}=G^{\prime} \times_{F^{\prime}} E^{\prime}$; and hence $H^{\prime}=G^{\prime}$, as desired.

Lemma 1.12. In the situation of Theorem 1.10, every normal algebraic subgroup $H \subset G^{\prime} \times{ }_{F^{\prime}} E^{\prime}$ which surjects to $\prod_{\sigma \in \Sigma} G \times_{F, \sigma} E^{\prime}$ is equal to $G^{\prime} \times_{F^{\prime}} E^{\prime}$.

Proof. Using descending induction on $i$ we will prove that $G_{\sigma, i} \subset H$ for all $\sigma \in \Sigma$ and $1 \leq i \leq q$. For $i=q$ the assertion is obvious, because $G_{\sigma, q}=1$. Let us assume the inclusion for $G_{\sigma, i+1}$ and abbreviate

$$
\operatorname{gr}_{i} H_{\sigma}:=\frac{H \cap G_{\sigma, i}}{G_{\sigma, i+1}} \subset \frac{G_{\sigma, i}}{G_{\sigma, i+1}} \stackrel{1.3}{\cong} \text { Lie } G \otimes_{F, \sigma} \mathbb{G}_{a, E^{\prime}} .
$$

By functoriality of the isomorphism 1.3 , the conjugation action of $G^{\prime}\left(E^{\prime}\right)$ on $G_{\sigma, i}$ corresponds to the adjoint representation of $G \times_{F, \sigma} E^{\prime}$ on the right hand side. As $H$ is a normal subgroup, all commutators between $H$ and $G_{\sigma, i}$ must lie in $H$. It follows that

$$
\left(\operatorname{Ad}_{h}-\mathrm{id}\right)(\operatorname{Lie} G) \otimes_{F, \sigma} \mathbb{G}_{a, E^{\prime}} \subset \operatorname{gr}_{i} H_{\sigma}
$$

for every $h \in H\left(E^{\prime}\right)$. Since $G$ is simply connected, it is known that the space of coinvariants of its adjoint representation is trivial (cf. [1], [2], or [5] Prop.1.11). On the other hand $E^{\prime}$ is algebraically closed, so by assumption $H\left(E^{\prime}\right)$ maps to a Zariski dense subgroup of $G \times_{F, \sigma} E^{\prime}$. Thus, as $h$ varies, the subgroups in 1.14 generate Lie $G \otimes_{F, \sigma} \mathbb{G}_{a, E^{\prime}}$. The inclusion in 1.13 is therefore an equality, and so we have $G_{\sigma, i} \subset H$.

At the end of the induction we have $G_{\sigma, 1} \subset H$ for all $\sigma \in \Sigma$. Combining this with the fact that $H$ surjects to $\prod_{\sigma \in \Sigma} G_{\sigma} / G_{\sigma, 1}$, we finally deduce $H=$ $G^{\prime} \times{ }_{F^{\prime}} E^{\prime}$, as desired. This proves Lemma 1.12 and thereby finishes the proof of Theorem 1.10 .

Remark 1.15. The analogue of Theorem 1.10 fails if $G$ is not simply connected and both $F / F^{\prime}$ and the universal central extension $\pi: \tilde{G} \rightarrow G$ are inseparable. The reason is that by Proposition 1.6 (b) the homomorphism $\mathcal{R}_{F / F^{\prime}} \varphi: \mathcal{R}_{F / F^{\prime}} \tilde{G} \rightarrow G^{\prime}$ is not surjective, so its image is a subgroup that makes $G^{\prime}$ not simple.

Corollary 1.16. If $G$ is semisimple and simply connected, then $G^{\prime}$ is perfect.

Proof. We may assume that $G$ is simple. Then $G$ is connected and non-commutative; hence so is $G^{\prime}$. The commutator group of $G^{\prime}$ is therefore non-trivial connected and normal, and by [11] Cor.2.2.8 it is "defined over $F$ " and thus smooth. By Theorem 1.10 it is therefore equal to $G^{\prime}$, as desired.

Theorem 1.17. If $G$ is simple isotropic and simply connected and $F$ is infinite, then $G^{\prime}$ is generated by split tori. 
Proof. By assumption there exists a closed embedding $\mathbb{G}_{m, F^{\prime}} \times_{F^{\prime}} F \cong \mathbb{G}_{m, F} \hookrightarrow$ $G$. The homomorphism $\mathbb{G}_{m, F^{\prime}} \rightarrow G^{\prime}$ corresponding to it by the universal property of the Weil restriction is again non-trivial; hence $G^{\prime}$ contains a non-trivial split torus. The algebraic subgroup of $G^{\prime}$ that is generated by all split tori in $G^{\prime}$ is therefore non-trivial. By construction it is normalized by $G^{\prime}\left(F^{\prime}\right)$, so by Theorem 1.7 it is normal in $G^{\prime}$. Being generated by smooth connected subgroups, it is itself smooth and connected by [11] Prop.2.2.6 (iii). By Theorem 1.10 it is therefore equal to $G^{\prime}$, as desired.

\section{Main results}

In the following we consider a connected semisimple group $G$ over a local field $F$. Let $\pi: \tilde{G} \rightarrow G$ denote its universal central extension. The commutator pairing $\tilde{G} \times \tilde{G} \rightarrow \tilde{G}$ factors through a unique morphism

$$
[,]^{\sim}: G \times G \rightarrow \tilde{G} .
$$

For any closed subgroup $\Gamma \subset G(F)$ we let $\tilde{\Gamma}^{\prime}$ denote the closure of the subgroup of $\tilde{G}(F)$ that is generated by the set of generalized commutators $[\Gamma, \Gamma]^{\sim}$.

Theorem 2.1. Let $F$ be a local field, and let $G$ be an isotropic connected simple semisimple group over $F$. Let $\Gamma \subset G(F)$ be a non-discrete closed subgroup whose covolume for any invariant measure is finite. Then $\tilde{\Gamma}^{\prime}$ is open in $\tilde{G}(F)$.

Before proving this, we note the following consequence (cf. [8], [3]).

Corollary 2.2. Under the assumptions of Theorem 2.1 we have $\tilde{\Gamma}^{\prime}=\tilde{G}(F)$. In particular, $\Gamma$ contains $\pi(\tilde{G}(F))$.

Proof. Since $G(F)$ is not compact and $\Gamma$ is a subgroup of finite covolume, this subgroup is not compact. Thus $\tilde{\Gamma}^{\prime}$ is normalized by an unbounded subgroup of $G(F)$, and it is open in $\tilde{G}(F)$ by Theorem 2.1. As in [6] Thm.2.2 one deduces from this that $\tilde{\Gamma}^{\prime}$ is unbounded. Let $\tilde{G}(F)^{+}$denote the subgroup of $\tilde{G}(F)$ that is generated by the rational points of the unipotent radicals of all rational parabolic subgroups. The Kneser-Tits conjecture, which is proved in this case (see [7] Thm. 7.6 or [10]), asserts that $\tilde{G}(F)^{+}=\tilde{G}(F)$. On the other hand, a theorem of Tits [9] states that every unbounded open subgroup of $\tilde{G}(F)^{+}$is equal to $\tilde{G}(F)^{+}$. Altogether this implies $\tilde{\Gamma}^{\prime}=\tilde{G}(F)$, as desired.

Proof of Theorem 2.1. In the case $\operatorname{char}(F)=0$ the proof in [8] $\S 2$ cannot be improved. It covers in particular the archimedean case. We will give a unified proof in the non-archimedean case, beginning with a few reductions.

Let $\Gamma^{\text {ad }}$ denote the image of $\Gamma$ in the adjoint group $G^{\text {ad }}$ of $G$. Then $\tilde{\Gamma}^{\prime}$ depends only on $\Gamma^{\text {ad }}$. On the other hand, all the assumptions in 2.1 are still satisfied for $\Gamma^{\mathrm{ad}} \subset G^{\mathrm{ad}}(F)$. Namely, since the homomorphism $G(F) \rightarrow G^{\mathrm{ad}}(F)$ is proper with finite kernel, the subgroup $\Gamma^{\mathrm{ad}}$ is still non-discrete and closed. On the other hand, as the image of $G(F)$ in $G^{\mathrm{ad}}(F)$ is cocompact, the covolume of $\Gamma^{\mathrm{ad}}$ in $G^{\mathrm{ad}}(F)$ 
is again finite. To prove the theorem, we may therefore replace $G$ by $G^{\text {ad }}$ and $\Gamma$ by $\Gamma^{\text {ad }}$. In other words, we may assume that $G$ is adjoint.

Next, since $G$ is connected simple and adjoint, it is isomorphic to $\mathcal{R}_{F_{1} / F} G_{1}$ for some absolutely simple connected adjoint group $G_{1}$ over a finite separable extension $F_{1} / F$. If $\tilde{G}_{1}$ denotes the universal covering of $G_{1}$, we then have $\tilde{G} \cong \mathcal{R}_{F_{1} / F} \tilde{G}_{1}$. By the definition of Weil restriction we have $G(F) \cong G_{1}\left(F_{1}\right)$ and $\tilde{G}(F) \cong \tilde{G}_{1}\left(F_{1}\right)$; and since $G$ is isotropic, so is $G_{1}$. Thus after replacing $F$ by $F_{1}$ and $G$ by $G_{1}$ we may assume that $G$ is absolutely simple.

For the next preparations note that $F$ is non-archimedean, so $G(F)$ possesses an open compact subgroup. Its intersection with $\Gamma$ is an open compact subgroup of $\Gamma$; let us call it $\Delta$. Let $\tilde{\Delta}^{\prime}$ denote the closure of the subgroup of $\tilde{G}(F)$ that is generated by the set of generalized commutators $[\Delta, \Delta]^{\sim}$.

We will study the relation between these subgroups and various Weil restrictions of $G$. Consider any closed subfield $F^{\prime} \subset F$ such that $\left[F / F^{\prime}\right]$ is finite. Note that in the case $\operatorname{char}(F)=0$ there is a unique smallest such $F^{\prime}$, namely the closure of $\mathbb{Q}$. But in positive characteristic the extension $F / F^{\prime}$ may be arbitrarily large and, what is worse, it may be inseparable.

Set $G^{\prime}:=\mathcal{R}_{F / F^{\prime}} G$ and $\tilde{G}^{\prime}:=\mathcal{R}_{F / F^{\prime}} \tilde{G}$, and let $\pi^{\prime}: \tilde{G}^{\prime} \rightarrow G^{\prime}$ be the homomorphism induced by $\pi$. From Proposition 1.6 we know that $\pi^{\prime}$ is not necessarily an isogeny. Identifying $G(F)$ with $G^{\prime}\left(F^{\prime}\right)$ via the universal property of the Weil restriction, we can view $\Gamma$ as a non-discrete closed subgroup of finite covolume of $G^{\prime}\left(F^{\prime}\right)$. Similarly, we can view $\tilde{\Delta}^{\prime}$ as a subgroup of $\tilde{G}^{\prime}\left(F^{\prime}\right)$.

Lemma 2.3. $\tilde{\Delta}^{\prime}$ is Zariski dense in $\tilde{G}^{\prime}$.

Proof. Let $H^{\prime} \subset G^{\prime}$ and $\tilde{H}^{\prime} \subset \tilde{G}^{\prime}$ be the Zariski closures of $\Delta$ and $\tilde{\Delta}^{\prime}$, respectively. By [11] Lemma 11.2.4 (ii) these groups are "defined over $F^{\prime \prime}$, i.e., smooth over $F^{\prime}$. The intersection of $\Delta$ with the identity component of $H^{\prime}$ is open in $\Delta$ and thus again an open compact subgroup of $\Gamma$. After shrinking $\Delta$ we may therefore assume that $H^{\prime}$ is connected. For any $\gamma \in \Gamma$ the subgroup $\gamma \Delta \gamma^{-1}$ is again an open compact subgroup of $\Gamma$, so it is commensurable with $\Delta$. Thus $\gamma H^{\prime} \gamma^{-1}$ is commensurable with $H^{\prime}$. Since $H^{\prime}$ is connected, they must be equal; hence $H^{\prime}$ is normalized by $\Gamma$. It is therefore also normalized by the Zariski closure of $\Gamma$.

Under the assumptions of 2.1, a theorem of Wang [12] implies that the Zariski closure of $\Gamma$ in $G^{\prime}$ contains all split tori of $G^{\prime}$. Thus, in particular, it contains the images under $\pi^{\prime}$ of all split tori in $\tilde{G}^{\prime}$. Since $G$ is simple isotropic, so is $\tilde{G}$; hence by Theorem 1.17 these tori generate $\tilde{G}^{\prime}$. It follows that $H^{\prime}$ is normalized by the image of $\tilde{G}^{\prime}$. By construction $\tilde{H}^{\prime}$ is the algebraic subgroup of $\tilde{G}^{\prime}$ that is generated by the image of the connected variety $H^{\prime} \times{ }_{F^{\prime}} H^{\prime}$ under [, ] ${ }^{\sim}$. It is therefore connected and normalized by $\tilde{G}^{\prime}$.

Since $\Gamma$ is non-discrete, the group $\Delta$ is not finite, and so $H^{\prime}$ is non-trivial. Let $H$ denote the image of $H^{\prime} \times{ }_{F^{\prime}} F$ under the canonical adjunction morphism $G^{\prime} \times F_{F^{\prime}} F \rightarrow G$. By construction $H$ is just the Zariski closure of $\Delta$ in $G$, so by the above arguments in the case $F^{\prime}=F$ it is normalized by the image of $\tilde{G}$. But $\pi: \tilde{G} \rightarrow G$ is surjective, so $H$ is a non-trivial connected normal subgroup of $G$. As $G$ is absolutely simple, this implies $H=G$. As $G$ is perfect, it follows that $\tilde{H}^{\prime} \times_{F^{\prime}} F$ surjects to $G$. 
All in all we now deduce that $\tilde{H}^{\prime}$ is a non-trivial connected smooth normal algebraic subgroup of $\tilde{G}^{\prime}$. By Theorem 1.10 this implies $\tilde{H}^{\prime}=\tilde{G}^{\prime}$, as desired.

Note that Lemma 2.3 in the case $F^{\prime}=F$ says that $\tilde{\Delta}^{\prime}$ is Zariski dense in $\tilde{G}$. In particular $\Delta$ is compact and Zariski dense in $G$, so we can apply [5] Main Theorem 0.2 . It follows that there exists a closed subfield $E \subset F$ such that $[F / E]$ is finite, an absolutely simple and simply connected semisimple algebraic group $\tilde{H}$ over $E$, and an isogeny $\tilde{\varphi}: \tilde{H} \times{ }_{E} F \rightarrow \tilde{G}$ with non-vanishing derivative, such that $\tilde{\Delta}^{\prime}$ is the image under $\tilde{\varphi}$ of an open subgroup of $\tilde{H}(E)$.

Lemma 2.4. $E=F$.

Proof. Via the universal property of the Weil restriction the isogeny $\tilde{\varphi}$ corresponds to a homomorphism $\tilde{\varphi}^{\prime}: \tilde{H} \rightarrow \mathcal{R}_{F / E} \tilde{G}$, which satisfies

$$
\tilde{\Delta}^{\prime} \subset \tilde{\varphi}^{\prime}(\tilde{H}(E)) \subset\left(\mathcal{R}_{F / E} \tilde{G}\right)(E)=\tilde{G}(F) .
$$

By Lemma 2.3 in the case $F^{\prime}=E$ we know that $\tilde{\Delta}^{\prime}$ is Zariski dense in $\mathcal{R}_{F / E} \tilde{G}$. It follows that $\tilde{\varphi}^{\prime}$ is dominant. This implies

$$
\operatorname{dim} \tilde{H} \geq \operatorname{dim} \mathcal{R}_{F / E} \tilde{G}=[F / E] \cdot \operatorname{dim} \tilde{G}=[F / E] \cdot \operatorname{dim} \tilde{H} ;
$$

hence $[F / E]=1$, as desired.

Lemma 2.5. $\tilde{\varphi}$ is an isomorphism.

Proof. As $\tilde{\varphi}$ is an isogeny between simply connected groups, it is an isomorphism if and only if it is separable. In characteristic zero this is automatically the case. (Since $d \tilde{\varphi} \neq 0$, this is actually true whenever $\operatorname{char}(F) \neq 2,3$ (cf. [5] Thm.1.7), but we do not need that fact.) So for the rest of the proof we may suppose that $p:=\operatorname{char}(F)$ is positive. Set $F^{\prime}:=\left\{x^{p} \mid x \in F\right\}$; then $F / F^{\prime}$ is an inseparable extension of degree $p$. Consider the induced homomorphism

$$
\tilde{\psi}:=\mathcal{R}_{F / F^{\prime}} \tilde{\varphi}: \mathcal{R}_{F / F^{\prime}} \tilde{H} \longrightarrow \mathcal{R}_{F / F^{\prime}} \tilde{G} .
$$

By construction it satisfies

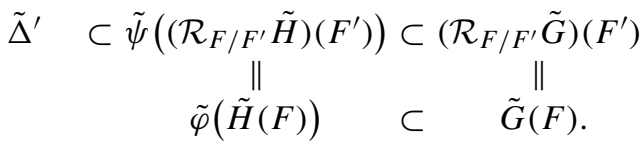

Since $\tilde{\Delta}^{\prime}$ is Zariski dense in $\mathcal{R}_{F / F^{\prime}} \tilde{G}$ by Lemma 2.3, we deduce that $\tilde{\psi}$ is dominant. So for dimension reasons it is an isogeny. Proposition 1.6 (b) now shows that $\tilde{\varphi}$ is separable, as desired.

Combining Lemmas 2.4 and 2.5, we now deduce that $\tilde{\Delta}^{\prime}$ is open in $\tilde{G}(F)$. Thus $\tilde{\Gamma}^{\prime}$ is open in $\tilde{G}(F)$, completing the proof of Theorem 2.1 .

Acknowledgements. The author would like to thank Gopal Prasad and Marc Burger for interesting conversations, and especially the former for suggesting a combination of the methods of [8] and [5] to obtain another proof of strong approximation in arbitrary characteristic. 


\section{References}

1. Hiss, G.: Die adjungierten Darstellungen der Chevalley-Gruppen. Arch. Math. 42, 408-416 (1984)

2. Hogeweij, G.M.D.: Almost Classical Lie Algebras I. Indagationes Math. 44, 441-460 (1982)

3. Margulis, G.A.: Cobounded subgroups in algebraic groups over local fields. Funkcional. Anal. i Priložen 11 (2), 45-57 (1977); Funct. Anal. Appl. 11(2), 119-128 (1977)

4. Oesterlé, J.: Nombres de Tamagawa et groupes unipotents en caractéristique $p$. Invent. Math. 78, 13-88 (1984)

5. Pink, R.: Compact subgroups of linear algebraic groups. J. Algebra 206, 438-504 (1998)

6. Pink, R.: Strong approximation for Zariski dense subgroups over arbitrary global fields. Comm. Math. Helv. 75, 608-643 (2000)

7. Platonov, V., Rapinchuk, A.: Algebraic Groups and Number Theory. Boston etc.: Academic Press 1994

8. Prasad, G.: Strong approximation for semi-simple groups over function fields. Annals of Math. 105, 553-572 (1977)

9. Prasad, G.: Elementary proof of a theorem of Bruhat-Tits-Rousseau and of a theorem of Tits. Bull. Soc. math. France 110, 197-202 (1982)

10. Prasad, G., Raghunathan, M.S.: On the Kneser-Tits problem. Comment. Math. Helv. 60(1), 107-121 (1985)

11. Springer, T.A.: Linear Algebraic Groups. Second Edition. Boston etc.: Birkhäuser, 1998

12. Wang, S.P.: On density properties of $S$-subgroups of locally compact groups. Annals of Math. 94, 325-329 (1971) 